INPLASY

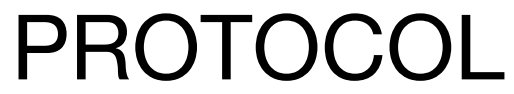

To cite: Neelakant et al.

Protocol for an updated systematic review and metaanalysis of prevalence of cognitive impairment in COPD patients. Inplasy protocol 202070120. doi:

10.37766/inplasy2020.7.0120

Received: 27 July 2020

Published: 28 July 2020

Corresponding author:

Hoong Wei Tan

HoongWeiTan@rcsi.com

Author Affiliation:

Department of Health

Psychology, Royal College of

Surgeons in Ireland (RCSI).

Support: None.

Review Stage at time of this submission: Preliminary

searches.

Conflicts of interest:

None.

\section{Protocol for an updated systematic review and meta-analysis of prevalence of cognitive impairment in COPD patients}

Neelakant, T1; Tan, HW2; Blackburne, M3; Doyle, F4.

Review question / Objective: What is the prevalence of cognitive impairment in COPD patients?

Condition being studied: Prevalence of cognitive impairment in COPD patients will be studied. Cognitive impairment is defined as scoring above threshold for impairment, as assessed via standardised tests such as Mini Mental State exam or similar questionnaires.

Information sources: Electronic databases. If data is missing authors will be contacted.

INPLASY registration number: This protocol was registered with the International Platform of Registered Systematic Review and Meta-Analysis Protocols (INPLASY) on 28 July 2020 and was last updated on 28 July 2020 (registration number INPLASY202070120).

\section{INTRODUCTION}

Review question / Objective: What is the prevalence of cognitive impairment in COPD patients?
Rationale: While a previous systematic review explored the prevalence of cognitive impairment in COPD (Torres-Sanchez et al.), it was flawed in that the search strategy was not comprehensive and the authors did not conduct a meta-analysis. 
We therefore aim to fill this gap in the literature by addressing these limitations.

Condition being studied: Prevalence of cognitive impairment in COPD patients will be studied. Cognitive impairment is defined as scoring above threshold for impairment, as assessed via standardised tests such as Mini Mental State exam or similar questionnaires.

\section{METHODS}

Search strategy: Medline, SCOPUS, CINAHL and PsychInfo.

Participant or population: People with COPD, who have been assessed with a standardised measure of cognitive impairment.

Intervention: None.

Comparator: Not applicable.

Study designs to be included: Observational studies.

Eligibility criteria: Only patients with COPD are included. Exclusion criteria are asthma and other pulmonary diseases.

Information sources: Electronic databases. If data is missing authors will be contacted.

Main outcome(s): Prevalence of cognitive impairment, as per standardised assessments (using recommended thresholds).

Additional outcome(s): None.

Data management: EndNote X9.

Quality assessment / Risk of bias analysis: Two reviewers will independently appraise the obtained studies using the JoannaBriggs Institute Critical Appraisal Tool. Discrepancies will be discussed with a third reviewer.

Strategy of data synthesis: Meta-analysis will be conducted using the metaprop command in Stata version 15. Prevalence of any cognitive impairment will be calculated as the primary analysis.

Subgroup analysis: Subgroup analysis will explore different cognitive impairments, such as perception, attention, memory and learning, executive function and abstract thinking, language, intelligence and general/global.

Sensibility analysis: We will consider sensitivity analyses depending on the cognitive assessment tool.

Language: English articles only will be considered.

Country(ies) involved: Ireland, Canada, Malaysia.

Keywords: Cognitive impairment, COPD, prevalence, meta-analysis.

Dissemination plans: The findings will be submitted to a peer reviewed journal and presented at conferences.

Contributions of each author:

Author 1 - Trisha Neelakant - Searches, manuscript drafting, data extraction.

Author 2 - Hoong Wei Tan - Searches, manuscript drafting, quality appraisal, data extraction.

Author 3 - Megan Blackburne - Quality appraisal, manuscript drafting, data extraction.

Author 4 - Frank Doyle - Conception, manuscript drafting, analysis. 